complicated initial febrile seizures and ES are also at greater risk of developing epilepsy

\title{
EPILEPSY AND COGNITIVE PERFORMANCE
}

Changes in left temporal interictal epileptiform activity during and after the performance of cognitive tasks are reported in an 18-year-old male with intractable complex partial seizures evaluated at the Radcliffe Infirmary, Oxford, UK. Epileptiform discharges were suppressed or enhanced depending on the nature and timing of the tasks. A posterior temporal spike focus occurred only during rest periods that followed verbal tasks. The mean left mid-to-anterior temporal spike count was halved during verbal tasks compared to the count during visuo-spatial tasks. A relatively low rate of right-sided temporal discharges was unchanged during or after the performance of tasks. (Boniface SJ et al. Changes in focal interictal epileptiform activity during and after the performance of verbal and visuospatial tasks in a patient with intractable partial seizures. I Neurol Neurosurg Psychiatry Feb 1994; $57: 227-228$ ). (Respond: Dr SJ Boniface, Dept of Clinical Neurophysiology, Radcliffe Infirmary, Woodstock Road, Oxford OX2 6HE, UK).

COMMENT. Activation or suppression of EEG focal spike discharges in relation to psychological testing may have a role in the medical treatment of certain children with reading and other learning disabilities as well as the assessment of patients before and after surgery for epilepsy.

\section{ANTIEPILEPTIC DRUGS}

\section{PHENYTOIN AND COGNITIVE-MOTOR PERFORMANCE}

Cognitive-motor function in 51 children with seizures well controlled with phenytoin (PHT) monotherapy was assessed in relation to drug concentration, seizure type, and time of medication at the Departments of Psychiatry and Pharmacology, Auckland University School of Medicine, Australia. Age ranged from 4 to 14 years, and performance was significantly better in older patients. Diagnosis (partial vs generalized epilepsy), PHT concentration levels, and change from trough to peak concentration days had little effect. Fluctuations in PHT as great as $50 \%$ had no or minimal effects on performance of tests in low therapeutic doses. (Aman MG, Werry JS et al. Effects of phenytoin on cognitive-motor performance in children as a function of drug concentration, seizure type, and time of medication. Epilepsia Jan/Feb 1994;35:172-180). (Reprints: Dr MG Aman, Nisonger Center, Ohio State University, 1581 Dodd Dr, Columbus, OH 43210).

COMMENT. Maintenance phenytoin monotherapy, at relatively low therapeutic levels, had negligible or no effects on cognitive motor function in a group of children with well controlled seizures.

Performance swings resulting from drug absorption and elimination were absent or minimal in this carefully monitored study. The importance of frequent determinations of phenytoin levels during evaluations of neuropsychological function in children is evident from the following report. 
In a special article on the "role of therapeutic drug monitoring in pediatric anticonvulsant drug dosing," Walson PD at Children's Hospital, Columbus, $\mathrm{OH}$ refers to a rapid phenytoin clearance and a first order (linear) rather than saturated kinetics observed in some children found to have unusually low serum drug levels despite doses as high as 18 $\mathrm{mg} / \mathrm{kg} /$ day. (Brain \& Dev 1994;16:23). The effects of rate and extent of absorption on the interpretation of phenytoin concentrations and cognitive function are often unappreciated. Various factors can modify phenytoin absorption in children, including the dose and stool frequency. Doses well tolerated in healthy children may become toxic if the patient is constipated. High dose phenytoin loading can affect glucose homeostasis, with possible changes in cognition. The hyperglycemic effect of phenytoin was first demonstrated in the Division of Neurology and Neurochemistry Laboratories at Children's Memorial Hospital, Chicago (Belton NR, Etheridge JE Jr, and Millichap JG. Effects of convulsions and anticonvulsants on blood sugar in rabbits. Epilepsia $1965 ; \underline{6}: 234)$.

\section{BRAIN NEOPLASMS}

\section{SURVEILLANCE SCANNING FOR MEDULLOBLASTOMA}

The value of surveillance scanning, compared to periodic history taking and physical examination, in detection of asymptomatic recurrent tumors was examined in 86 children with posterior fossa medulloblastoma followed regularly between 1980 and 1991 at the Children's Hospital of Philadelphia. Recurrences were diagnosed in 23 (27\%); 4 were detected by scanning only and 19 were associated with symptoms that developed at a median of 4 months after the last scan. No patient survived after a recurrence. (Torres CF et al. Surveillance scanning of children with medulloblastoma. N Engl I Med March 31, 1994;3ㅜㅇ:892-5). (Reprints: Dr Beverly J Lange, Division of Oncology, Dept of Pediatrics, Children's Hospital of Philadelphia, University of Pennsylvania School of Medicine, Philadelphia, PA 19104).

COMMENT. The authors conclude that surveillance scanning in children with medulloblastoma has limited clinical value. General anesthesia was required for MRI in $10 \%$ of children under 4 years of age, and sedation was requested or required by most children under the age of 7 years. More frequent scanning has the disadvantages of morbidity from sedation or anesthesia and increased cost, and earlier detection of recurrence is unlikely to change the outcome. Regular clinical evaluation and scanning are recommended to determine response and change in status after therapy. After a maximal response is achieved, scanning should be based on clinical symptoms and signs. These investigators had reached these conclusions more than 18 months ago and had presented their findings in Oct 1992: Torres C et al. Ann Neurol Sept 1992; $\underline{32}$ :458(abstract). (see Ped Neur Briefs Dec 1992;ㅁ:94). 